\title{
Hypoparathyroidism-sensorineural deafness-renal disease syndrome
}

INSERM

\section{Source}

INSERM. (1999). Orphanet: an online rare disease and orphan drug data base.

Hypoparathyroidism-sensorineural deafness-renal disease syndrome. ORPHA:2237

Hypoparathyroidism-sensorineural deafness-renal disease syndrome is a rare, clinically heterogeneous genetic disorder characterized by the triad of hypoparathyroidism $(\mathrm{H})$, sensorineural deafness (D) and renal disease (R). 